\title{
Monte Carlo model for neutrino-nucleus interactions: past, present and future
}

\author{
Sergey M. Eliseev ${ }^{1, *}$ and Bekhzad S. Yuldashev ${ }^{2, * *}$ \\ ${ }^{1}$ Joint Institute for Nuclear Research, 141980, Dubna, Moscow Region, Russia \\ ${ }^{2}$ Institute of Nuclear Physics, Uzbekistan Academy of Sciences, Tashkent, 100214, Uzbekistan
}

\begin{abstract}
Quantum Chromodynamics (QCD) is the correct theory of strong interactions. The main direction of investigations in physics of elementary particles and nuclear physics is testing of QCD. QCD predicts that at high energy density there will be a transformation from ordinary nuclear matter to a plasma of free quarks and gluons, the Quark-Gluon Plasma (QGP). In order to reach new knowledge of QCD from the interaction of relativistic heavy ions, one needs directly comparable data sets from systems of various sizes, different energies and different experimental probes. Lepton-nucleus scattering provides a nontrivial possibility to study space-time evolution of jets inside the nuclear matter. Using QCD-inspired time dependent cross sections for pre-hadrons we have introduced a space-time model for propagation and hadronization of quark and gluon jets in the nuclear matter in DIS. The aim of this work is to examine a multiproduction process of charged-current deep inelastic $v_{\mu}$-nucleus and nuclear emulsion scattering and estimate quantitatively the value of the formation time. These studies may help to explain the jet quenching in heavy ion collisions. In conclusion, the role of neutrino generators in modern neutrino experiments with nuclear targets will be discussed.
\end{abstract}

\section{Introduction}

The interactions of relativistic heavy ion collisions provide the necessary conditions to investigate many problems: manifestations of quark and gluon degrees of freedom in nuclei, phase transition between ordinary matter and hadron matter of high energy density, and so on $[1,2]$. The multiparticle production in ultra-relativistic heavy ion collisions is an important tool to study the perturbative as well as non-perturbative nature of QCD. This opportunity will be explored with the Nuclotron (JINR, Dubna), and with the accelerators, the BNL Relativistic Heavy Ion Collider (RHIC) and CERN Large Hadron Collider (LHC). Enormous data have been collected by RHIC and LHC from various types of collisions over a wide range of center-of-mass energy. The hot and dense matter produced in relativistic heavy ion collisions provides the condition to investigate the new state of matter of QCD, the so-called quark-gluon plasma (QGP). However, the space-time evolution of QGP and its hadronization are open questions in the investigation of this process [1,2]. Although it is generally believed that QCD is the fundamental theory of strong interactions, quantitative tests have

\footnotetext{
*e-mail: selis@theor.jinr.ru

**e-mail: Bekhzad.Yuldashev@cern.ch
} 
so far been restricted to the high momentum transfer domain where perturbative methods based on asymptotic freedom can be used. Tests of the confining non-perturbative aspects of the theory are qualitative and indirect. In addition, the question of fundamental importance in QCD is the hadronization-mechanism which converts quark and gluon quanta into integrally-charged final state hadrons.

Studies of QCD in the space-time domain have attracted attention over the past decade due to the availability of several new data. In the case of cold nuclei, the time development of fundamental processes can be studied in detail using the well understood nuclear system as a spatial analyzer. In the case of relativistic heavy ion collisions, the same processes can be used as tools to explore the nature of the hot dense matter.

In various models of interactions of particles with nuclei the interaction is interpreted as a sequence of independent collisions of the incident particle or its constituents with single nucleons inside the nucleus. In addition, the products of each projectile collision may interact with other nucleons of the nucleus and produce more particles. This process is called intra-nuclear cascading. Analyzing the data on multiparticle production in hadron-nucleus interactions at high energy it has turned out that cascading of secondaries is considerably lower than the expected one under the assumption that a secondary pion is able to interact at once after it has been produced in a nucleon-nucleon collision. And this has been attributed by the effect of the formation time of secondary particles.

\section{The model}

Collisions of particles and nuclei at high energies usually produce many hadrons and their production is a typical process where nonperturbative phenomena are involved. In inelastic scattering experiments the reaction products are hadronized long before they reach the detector. By using elementary nucleon targets one cannot obtain information on the spacetime picture of hadronization. A simple estimate of the hadron formation proper time via the hadronic radius $r_{h}$ gives hadron formation lengths of the order $\gamma \cdot r_{h}$ in the laboratory frame. At high energies the Lorentz factor $\gamma$ leads to such hadron formation lengths which can easily exceed typical nuclear dimensions. By utilizing nuclear targets one, therefore, has the unique opportunity to investigate the final-state interactions of the pre-hadronic system and study the dynamics of the hadronization process.

In various respects the lepton-induced reactions differ fundamentally from the hadron induced ones. To a good approximation the only mechanism for particle multiplication in this case is intra-nuclear cascading, which therefore can be investigated in a direct and clear way. This is in contrast to the hadron-induced reactions, where multiple collisions of the projectile form another important mechanism for particle production. In addition, leptons probe the full nuclear volume, but hadrons probe only the nuclear surface. Hadron production in deep inelastic lepton-nucleus scattering (DIS) provides an ideal tool to investigate the space-time evolution of hadron formation. The last few years have witnessed a large revival of interest to inclusive DIS (HERMES, NOMAD and other collaborations) [3-5].

The aim of this work is to examine a multiproduction process of charged-current deep inelastic $v_{\mu}$ - emulsion and $v_{\mu}-{ }^{20} \mathrm{Ne}$ scattering and estimate quantitatively the value of the formation time. We have developed a cascade model of multiproduction of neutrino-nuclei interactions. The model describes a branching process of the evolution of the parton jet (up to hadronization) in the atomic nucleus.

We assume that the interaction between the incident lepton and a target nucleus takes place in a lepton-nucleon interaction. The nucleus is excited by a series of collisions between secondaries (produced in the first lepton-nucleon interaction) and the intra-nuclear nucleons. This process continues until all secondaries escape the target nucleus. Part of the energy is 
spread through the nucleus to produce a fully-equilibrated nucleus which then decays statistically. The process of generation of particles is simulated by the Monte Carlo method.

The characteristics of the partons from the neutrino-nucleon interaction are taken from experiments with free nucleons [6-8]. (The parton spectra are assumed to be the same as the hadronic one. This approach is based on the concept of Local Parton Hadron Duality). The multiplicities of neutral particles (pions) are taken from the experiment. These multiplicities for the $v \mathrm{p}$ and $v \mathrm{n}$ interactions are, respectively, as follows:

$$
n(v p)_{\pi^{-}}=0.72+0.22 n_{\pi^{-}}, \quad n(v n)_{\pi^{-}}=0.14+0.73 n_{\pi^{-}},
$$

where $n_{\pi^{-}}$is the multiplicity of negatively charged particles. The angular and momentum distributions of neutral particles are assumed to be the same as those of positively and negatively charged particles. The characteristics of the interactions of the produced particles with nucleons in the nucleus are taken from experiments with free nucleons.

Since the interaction cross section of neutrino with a nucleon is small, we assume that the neutrino can interact with any nucleon of the nucleus with equal probability. Following the experiments and the quark parton model, we take into account that the interaction cross section of neutrino with a neutron is twice that with a proton, $\sigma(v+n) / \sigma(v+p) \approx 2$.

The spatial distribution of nucleons in the rest frame of the nucleus is sampled randomly due to the Woods - Saxon distribution.

The nuclear density is taken from the Woods-Saxon expression

$$
\rho(r) \sim 1 /\left(1+e^{(r-c) / \alpha}\right)
$$

with the parameters $\alpha=0.57 \mathrm{fm}$ and $c=1.19 A^{1 / 3}-1.61 A^{-1 / 3} \mathrm{fm}$. Moreover, a threedimensional geometry is used in our calculations.

Nuclear effects such as the Fermi motion and Pauli blocking are all taken into consideration. We describe the nucleus in the framework of the Fermi gas model. After introducing the Fermi motion and the Pauli principle the only remaining parameter necessary to simulate the complete intra-nuclear cascade is the formation time $l_{f}$ of secondaries. The space-time characteristics of lepton-nucleon interactions inside the target nucleus were taken into consideration. After time $\tau$ from the intra-nuclear collision the time-dependent cross section $\sigma_{h N}$ for the next collision of a secondary particle with a nucleon inside the nucleus is given by [9-11]

$$
\sigma_{h N}=\sigma_{h N}^{\exp }\left(1-e^{-\tau / \tau_{0}}\right)
$$

where $\tau$ is the time from the moment of production of this particle in the previous collision and $\sigma_{h N}^{\text {exp }}$ is the experimentally determined total interaction cross section of a hadron with a free nucleon at the corresponding energy of the secondary particle. Thus, only after a relatively long time $\tau$ the cross section of intra-nuclear interaction reaches the value $\sigma_{h N}^{\exp }$. In our equation, the parameter $\tau_{0}$ is a certain characteristic corresponding to the formation time of the secondary generated hadron.

The equation for $\sigma_{h N}$ can be rewritten in the form with the formation time parameter $l_{f}$ in the system of moving particles. The time $\tau$ is equal to $l / \beta \gamma$ where $l$ is the secondary particle range inside the nucleus(the distance traversed by the secondary particle of velocity $\beta(=v / c)$ from its generation point to the point of interaction with a nuclear nucleon. The Lorentz factor of the generated particle is $\gamma=\left(1-\beta^{2}\right)^{-1 / 2}$. The equation for $\sigma_{h N}$ can be rewritten in the form

$$
\sigma_{h N}=\sigma_{h N}^{\exp }\left(1-e^{-l / l_{f} \beta \gamma c}\right)
$$

The theoretical results for neutrino-nucleus interactions and their comparison with experimental data will be described below. 


\section{Results and discussion}

In this section, we present the theoretical results for neutrino-nuclear emulsion and neutrino $-{ }^{20} \mathrm{Ne}$ interactions and compare them with experimental data. (The full list of the references of experimental data can be found in $[12,13]$ and references cited therein.)

In the high-energy nuclear interactions there are also particles emitted backwards which have energies not allowed by the kinematics of collisions on a free nucleon. Within our cascade models, the production of particles in the kinematically forbidden region can be seen as the result of multiple scattering and of interactions of the secondary hadrons, produced in the primary interaction of $v_{\mu}$ with an intra-nuclear nucleon.

Fig. 1 shows the spectrum of the protons emitted backwards, with respect to the beam direction, which have energies not allowed by the kinematics of collisions on a free and stationary nucleon. The dotted curve (with big slope) demonstrates the production of protons with $\left.P^{2} \leq 0.2(\mathrm{GeV} / \mathrm{c})^{2}\right)$. The mechanism of this slow proton production is a evaporation process of the residual nuclei excited at the stage of propagation of jets in the nuclei. It is worth noting that in our approach the evolution of quark-gluon jets in nuclei in the framework of our model is accompanied by the nucleon emission at backward angles and momentum $\geq 300 \mathrm{MeV} / \mathrm{c}$ (cumulative protons). The spectrum of these protons is depicted by the solid curve in Fig. 1.

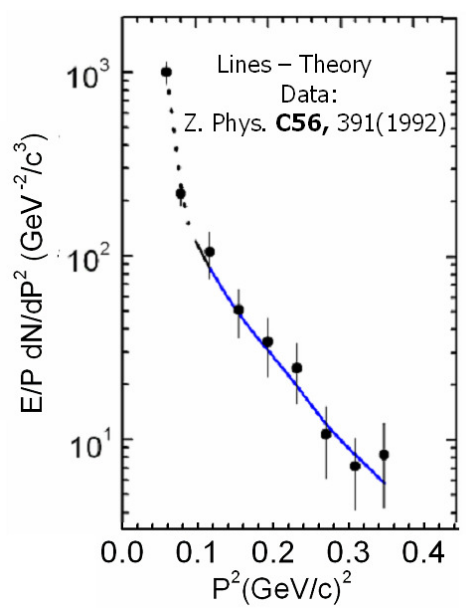

Figure 1. Invariant momentum distributions for backward emitted protons in interactions of neutrinos with nuclei in nuclear emulsion

These Cumulative Protons (CP) were observed in deep inelastic charged-current neutrinonuclear emulsion interactions [12]. The experimental multiplicity of CP $0.33 \pm 0.07$ is in good agreement with the calculated one equal to 0.29 . The effect of intra-nuclear absorption of particles (pions) by intra-nuclear 'deuterons' is well known in the theory of nuclear reactions at intermediate energy. This process is essential only for the slow pions (of energy $\leq 1 \mathrm{GeV}$ ). At high-energy beams this effect is only a few percent. As a result, the existing experimental data on CP from DIS of neutrino on nuclei can be interpreted, see the solid line in Fig. 1.

Below, we present our theoretical results for multiplicity of charged particles produced in neutrino-nucleus interactions and their comparison with experimental data. 
The multiparticle production in high-energy collisions is an important tool to study the perturbative as well as non-perturbative nature of QCD. Charged particle multiplicity is basic and general observables in modern collider experiments, which naturally contains the information on both: soft QCD processes and hard scattering.

In Tab. 1 we present the multiplicities of the charged hadrons produced in charge-current neutrino $-{ }^{20} \mathrm{Ne}$ interaction. A comparison between our theoretical calculations and the experimental data has clearly shown that:

1. The multiplicities of particles from $v_{\mu}-{ }^{20} \mathrm{Ne}$ interactions (calculated in our model) depend on the formation time $l_{f}$.

2. The model based on simple rescattering $\left(l_{f}=0\right)$ can not describe particle production.

3. The formation time of pions in the hadron rest frame. $l_{f}(\pi) \approx 0.5 \mathrm{fm} / \mathrm{c}$. 4. Formation time of protons, $l_{f}(p) \ll l_{f}(\pi)$. This is consistent with the QPM in which a difference in the fragmentation of quarks and diquarks is assumed. (Diquarks $u d$ are produced from the interaction of $v_{\mu}$ with a neutron of the nucleus; a proton is formed when diquark $u d$ picks up $u$ quark from the vacuum).

Table 1. The multiplicities of charged hadrons produced in the charge-current neutrino $-{ }^{20} \mathrm{Ne}$ interaction: the theory vs. experimental data. $\left({ }^{*}\right)$ for $\pi^{ \pm}$mesons and protons with $p_{p} \geq 1 \mathrm{GeV} / \mathrm{c},\left({ }^{* *}\right)$ for protons with $0.35 \leq p_{p} \leq 0.8 \mathrm{GeV} / \mathrm{c}$. The experimental data are taken from $[6-8,13]$

\begin{tabular}{|c|c|c|c|c|c|}
\hline \hline Particles & Experiment & Experiment & \multicolumn{3}{|c|}{ Calculations on our model } \\
\cline { 4 - 6 } & E172 & E546 & $l_{f}=0$ & $l_{f}=0.2 \mathrm{fm} / \mathrm{c}$ & $l_{f}=0.5 \mathrm{fm} / \mathrm{c}$ \\
\hline$<n\left(\pi^{-}\right)>$ & $1.37 \pm 0.04$ & $1.36 \pm 0.03$ & $1.53 \pm 0.02$ & $1.32 \pm 0.01$ & $1.41 \pm 0.01$ \\
\hline$<n^{ \pm}>$ & $3.98 \pm 0.11$ & $4.20 \pm 0.06$ & $5.68 \pm 0.02$ & $4.95 \pm 0.02$ & $4.16 \pm 0.02$ \\
\hline$<n_{p}>$ & & $0.33 \pm 0.01$ & $0.36 \pm 0.01$ & $0.30 \pm 0.01$ & $0.25 \pm 0.01$ \\
\hline \hline
\end{tabular}

The secondary particles in high-energy experiments with nuclear emulsion have been divided into three classes $[12,13]$. The first class includes relativistic or shower particles (sparticles) of velocity $\beta>0.7$. These particles are almost all charged pions of kinetic energy $\geq 60 \mathrm{MeV}$ and fast protons of kinetic energy $\geq 400 \mathrm{MeV}$. The second class consists of grey track particles (g-particles) of range $0.23 \leq \beta \leq 0.7$. They are mainly protons in the energy range of $27-400 \mathrm{MeV}$. The third class is the black track particles (b-particles). They are low energy particles emitted mainly due to evaporation.

Table 2. The average multiplicities of s- and g-particles, produced in charged-current $v_{\mu}$-nuclear emulsion interaction

\begin{tabular}{cccc}
\hline \multirow{2}{*}{$\begin{array}{c}\text { Experimental } \\
\text { data }\end{array}$} & \multicolumn{3}{c}{ Calculations of the model } \\
\cline { 2 - 4 } & $l_{f}=0.2 \mathrm{fm} / \mathrm{c}$ & $l_{f}=0.5 \mathrm{fm} / \mathrm{c}$ & $l_{f}=1 \mathrm{fm} / \mathrm{c}$ \\
\hline$N_{s} 5.28 \pm 0.26$ & $5.60 \pm 0.04$ & $5.12 \pm 0.03$ & $4.08 \pm 0.02$ \\
$N_{g} 1.33 \pm 0.15$ & $1.71 \pm 0.02$ & $1.35 \pm 0.02$ & $0.82 \pm 0.01$ \\
\hline
\end{tabular}

In Tab. 2 we show the average multiplicities of s- and g-particles produced in chargedcurrent $v_{\mu}$-nuclear emulsion interaction, compared with the corresponding quantities calculated on our model at different values of $l_{f}$.

One can see from this Tab. 2 that the experimental and calculated data at $l_{f}=0.5 \mathrm{fm}$ are in good agreement. 
In Tab. 3 we compare the calculated multiplicities of $g$ - and $b$-particles accompanied by a different number $k$ of cumulative protons with data.

Table 3. The average multiplicity of $N_{g}$ and $N_{b}$ particles associated with a different number $k=0,1$ and $k \geq 2$ of final state cumulative protons. The formation time is $l_{f}=0.5 \mathrm{fm} / \mathrm{c}$. The experimental data are given in parentheses

\begin{tabular}{cccc}
\hline$k$ & $N_{g}(\vartheta \leq \pi)$ & $N_{g}(\pi / 2 \leq \vartheta \leq \pi)$ & $N_{b}$ \\
\hline 0 & $1.1(1.4 \pm 0.1)$ & $1.1(1.4 \pm 0.1)$ & $3.9(4.4 \pm 0.2)$ \\
1 & $2.8(3.0 \pm 0.3)$ & $1.8(2.0 \pm 0.3)$ & $5.2(5.4 \pm 0.6)$ \\
$\geq 2$ & $5.6(5.6 \pm 0.5)$ & $3.0(3.1 \pm 0.6)$ & $9.0(10 . \pm 1.0)$ \\
\hline
\end{tabular}

In summary, the parton propagation in nuclear matter and hadron production in neutrinonuclei deep inelastic scattering have been studied by using the concept of time-dependent cross section [9-11]. The results of the calculations are compared with the experimental data and the distance of parton hadronization, $l_{f}$, in the nucleus is determined. Note that the formation time of particles plays the leading role in various present-day investigations of different nuclear reactions. In conclusion, the role of neutrino-nuclei generators in modern neutrino and other nuclear and high-energy experiments is discussed in [5, 14-17].

\section{References}

[1] A.W. Thomas, EPJ Web Conf. 123, 01003 (2016)

[2] C. Nattrass, EPJ Web Conf. 172, 05010 (2018)

[3] K. Gallmeister, U. Mosel, Nucl. Phys. A 801, 68 (2008)

[4] W.K. Brooks, H. Hakobyan, Nucl. Phys. A 830, 361c (2009)

[5] U. Mosel, CERN Courier 57, 23 (2017)

[6] T.Y. Burnett et al., Phys. Lett. B 77, 443 (1978)

[7] R.G. Ammar et al., JETP Lett. 49, 219 (1989)

[8] P. Astier et al. (NOMAD), Nucl. Phys. B 609, 255 (2001)

[9] S.M. Eliseev, B.S. Yuldashev, JINR P2-82-323, 8 (1982)

[10] S.M. Eliseev, B.S. Yuldashev, Sov. J. Nucl. Phys. 40, 601 (1984)

[11] A, El- Naghy, S.M. Eliseev, J. Phys. G 16, 39 (1990)

[12] M. Dayon et al. (FNAL-564), Z. Phys. C 56, 391 (1992)

[13] B.S. Yuldashev, Interaction Of Particles With Nuclei At High Energies (FAN, Tashkent, 1981) 74

[14] W. Cassing, K. Gallmeister, C. Greiner, Nucl. Phys. A735, 277 (2004)

[15] Y.B. He, J. Hufner, B.Z. Kopeliovich, Phys. Lett. B 477, 93 (2000)

[16] T. Falter, W. Cassing, K. Gallmeister, and U. Mosel, Phys. Rev. C 70, 054609 (2004)

[17] R. Bellwied, C. Markert, Phys. Lett. B 690, 208 (2010) 